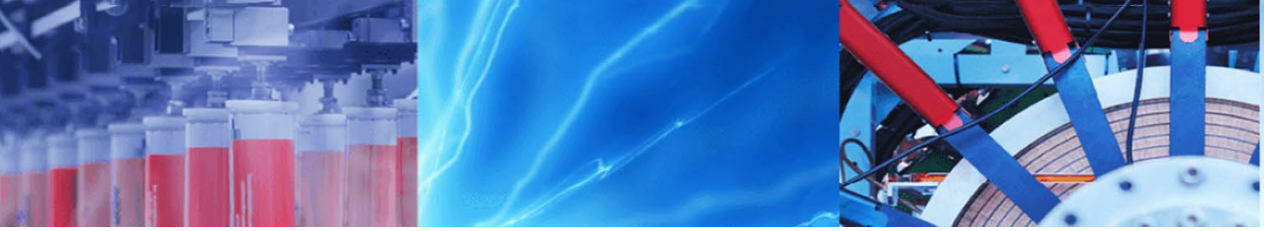

Research Article

\title{
Intelligent hybrid approaches for ensuring better prediction of gas-assisted EDM responses
}

\author{
Nishant K. Singh ${ }^{1} \cdot$ Rajeev Kumar Upadhyay ${ }^{1} \cdot$ Yashvir Singh $^{2} \cdot$ Abhishek Sharma $^{3}$
}

Received: 23 December 2019 / Accepted: 30 March 2020 / Published online: 16 April 2020

(c) Springer Nature Switzerland AG 2020

\begin{abstract}
The present research work explores the implementation of three smart hybrid predictive models based on the adaptive neuro-fuzzy inference system (ANFIS), ANFIS and genetic algorithm (GA), and ANFIS and particle swarm optimization (PSO). All such strategies have been used to determine and compare machining key elements including material removal rate (MRR) and surface roughness (SR) during the gas-assisted electrical discharge (GAEDM) process. In this study, inert gas-based EDM with a multi-hole rotating tool has been carried out. In this experimentation, pulse-on time, peak current, duty cycle, electrode rotation, and gas discharge pressure were selected as input factors. The proposed method is to upgrade ANFIS with GA and PSO techniques. The GA and PSO algorithms are used to enhance the accuracy of the ANFIS model. The models have been trained, tested, and validated with observational results. Statistical techniques were applied to assess the effectiveness of the predictive capability models established through the ANFIS, ANFIS-GA, and ANFIS-PSO techniques. The actual and predicated estimates of MRR and SR of the GAEDM, obtained by ANFIS, ANFISGA, and ANFIS-PSO, were observed to be as per one another. In addition, the ANFIS-PSO framework proved to be even more responsive when compared with the ANFIS and the ANFIS-GA system. In particular, the assertion of this work is that modified algorithms such as ANFIS-GA and ANFIS-PSO are an efficient and productive approach to accurate EDM response estimation.
\end{abstract}

Keywords ANFIS · ANFIS-GA · ANFIS-PSO - EDM · Soft-computing · Algorithms

\section{Introduction}

Electrical discharge machining (EDM) is a method of material processing and is commonly included in work that requires accuracy and precision. It has a variety of uses in the molding, die-making, and machining of parts with complicated profiles. The procedure uses heat energy to machine hard and stiff components having an electrical conductivity [1]. Moreover, the major inconvenience encountered during EDM is the removal eroded materials from the electrodes passage. The agglomerations of disintegrated maters in the discharge gap cause an arcing.
It results in increase machining time and inferior surface finish of the machined components [2]. Owing to the unpredictability and complexity of non-traditional manufacturing methods, classical modeling techniques cannot correctly predict the response. As a result, researchers have introduced numerous soft computing approaches that are prevalent in predicting process output due to their superior ability of learning from measured data, to illustrate the interaction impact of input factors in order to evaluate the output of the process with the desired result.

Diverse modeling techniques have been suggested during the contemporary times for the development of models

$\triangle$ Nishant K. Singh, nishant.singh78@gmail.com|'Department of Mechanical Engineering, Hindustan College of Science and Technology, Mathura, India. ${ }^{2}$ Department of Mechanical Engineering, Graphic Era Deemed To Be University, Dehradun, Uttarakhand, India. ${ }^{3}$ Department of Mechanical Engineering, GL Bajaj Institute of Technology and Management, Gr. Noida, India. 
for EDM process to correlate a link between process factors and the significant responses, for instance, surface roughness and material removal rate. In last couple of decades, researchers have applied artificial neural network technique extensively to develop the predictive models to evaluate the significant process parameters of EDM [3-6]. Panda [7] applied neuro-grey technique for modeling of important EDM output such as SR, MRR, and microhardness. The author suggested that developing models predict the process output more precisely in comparison with the statistical models. Substantial research has been conducted to combine the artificial neural network technique with the Taguchi method in order to optimize and develop an accurate surface response model [8-10]. During the experimental exploration of EDM using carbon nano-tube mixed dielectric, Prabhu et al. [11] applied the ANFIS approach to predict the surface finish of the machined specimen. The finding indicates that the model prediction is in good agreement with the value of the experiment. In order to predict the surface finish more precisely, Kar et al. [12] integrate the fuzzy logic with the Taguchi method in order to optimize process factors that have a particular impact on the electro-discharge coating operation. In this headway, Unune et al. [13] combine the ANN-RSM methodology to develop models that can predict the process output of the electro-discharge grinding process. In an other study, Prakash et al. [14] used hybrid Taguchi-RSM techniques to model the powder-mixed EDM responses and optimized process factors incorporating a non-dominated genetic algorithm strategy. In this process, Srivastava et al. [15] develop predictive models by integrating ANN and genetic algorithm together to accurately predict the output of the electro-discharge grinding process. The sorting recommended that the anticipated and exploratory values are compatible. More recently, Majumder and Maity [16] proposed a hybrid model based on the General Neural Regression Network (GRNN) and Multiple Regression Analysis (MRA) to predict important EDM responses. Authors noticed that the GRNN model prediction is more accurate compared to the MRA model. Singh and Sharma [17] applied hybrid grey-fuzzy-ANFIS techniques to measure the performance of powder-mixed EDM. They perform a comparative study of these two techniques, finding revealed that the proposed approaches can prove to be useful for obtaining the optimum parameters of multi-performance characteristics of PMEDM. Roy et al. [18] proposed hybrid techniques to optimize EDM responses. Authors integrated the fuzzy AHP and fuzzy TOPSIS strategy to get the optimal values of process factors. In their exploration, Teimouri et al. [19] used an adaptive neuro-fuzzy inference system to model the significant process responses of wire-EDM. Further, they applied an artificial bee colony $(A B C)$ algorithm in connection with ANFIS to optimize the WEDM responses. The confirmatory experiments verified that the proposed model predict the responses accurately. Recently, Singh et al. [20] performed a comprehensive analysis of predictions based on the ANN, ANFIS, and RSM approach to anticipate the MRR and SR during gas-assisted EDM. Finding revealed that the model based on ANFIS provides more correct predictions compared to other suggested techniques.

From the above discussion, it can be observed that the application of compressed gas in die-sinking EDM can improve MRR and surface roughness and results in better machinability. Although the number of works was reported in the literature using ANN and ANFIS techniques to measure the performance of EDM responses such as EWR, SR, and MRR, only a handful studies are available using the GA and ANFIS models. In addition, no research work about the use of hybrid GA-ANFIS and GA-Particle swan optimization (PSO) is documented, together in the assessment of EDM responses, and the current study is clearly innovative. No work on a comparative study of the EDM response between the hybrid ANFIS, ANFIS-GA, and ANFIS-PSO models has been revealed to the best of author's knowledge. Thus, the development of appropriate models for predicting EDM output responses accurately needs to be essential. It was perceived that hybrid soft computing techniques can provide a correct and exact prediction of EDM responses and proposed models can be utilized for industrial practice. This was the guiding force for the exploration to perform an appropriate modeling of the EDM response through the use of a revolutionary hybrid approach. The technique suggested is primarily focused on the assimilation of ANFIS-GA and ANFIS-PSO.

In this work, compressed inert (helium) gas is supplied in discharge gap in die-sinking EDM to improve the flushing effectiveness which results in better process performance. In order to forestall the oxidation reaction, an odd of flame and risks during the EDM machining compressed helium gas was utilized. An RSM-based matrix was used for the experimental design. In view of the acquired outcomes, models based on ANFIS, ANFIS-GA, and ANFISPSO were designed to measure the influence of distinct machining parameters on substantial EDM reactions in the Gas-Assisted Electrical Discharge Machining (GAEDM) process. The potency of the proposed models in anticipation of EDM performance was analyzed. This kind of research will help to develop a realistic model for the replication of the EDM process.

\section{Materials and methods}

\subsection{Tool and specimen material details}

The experiment was undertaken on D3 die steel as a workpiece using copper as an electrode material. 
The rectangular shape $\left(20 \times 15 \times 15 \mathrm{~mm}^{3}\right)$ specimen is utilized during the experimental work. A multi-hole tube as a tool was utilized to supply high-velocity gas through the electrode. The chemical compositions of the chosen specimens are depicted in Table 1. The hardness of the material is constant during the test (50HRC).

\subsection{Tool design}

The flow chart of the technique adopted is shown in Fig. 1. Appropriate tool geometry was specified in order to make sure smooth compressed gas flow. The use of the multi-hole electrode ensured the provision of gas stream flow, whereas the supply of compressed gas was provided through arc opening. In comparison with the tubular electrode, the multi-hole electrode has several
Table 1 Chemical ingredient of the workpiece

\begin{tabular}{lllllll}
\hline $\mathrm{Cr}$ & $\mathrm{C}$ & $\mathrm{Si}$ & $\mathrm{Mn}$ & $\mathrm{P}$ & $\mathrm{S}$ & $\mathrm{Fe}$ \\
\hline 10.05 & 2.30 & 0.40 & 0.30 & 0.05 & 0.03 & Rest \\
\hline
\end{tabular}

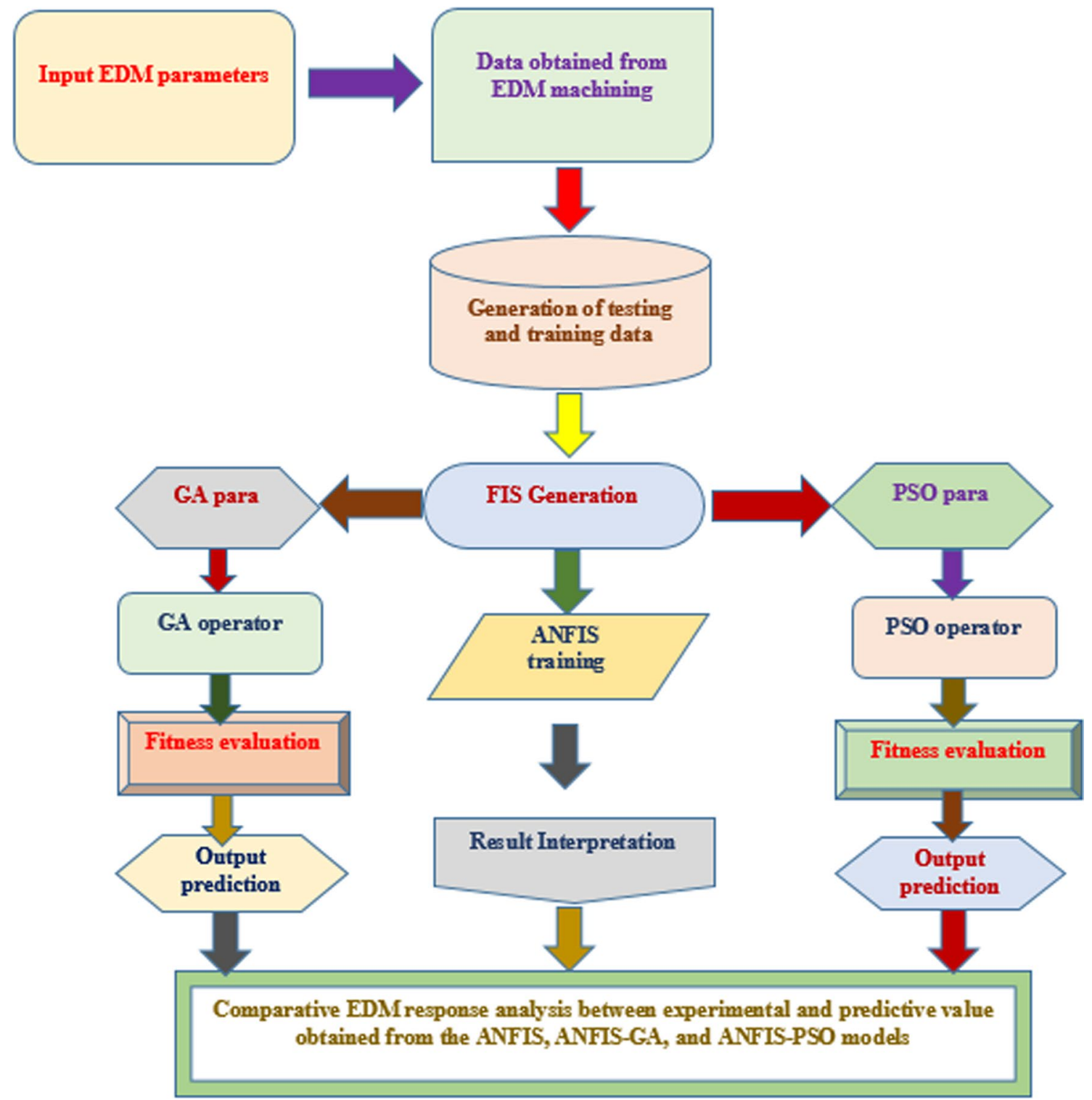

Fig. 1 Flow chart of adopted methodology 


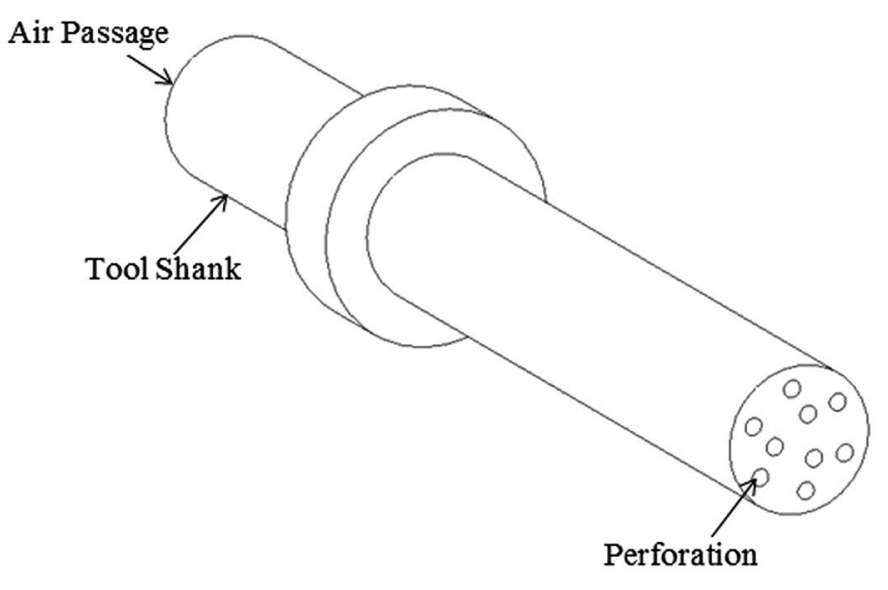

(a)



(b)

Fig. 2 Simplified illustration of a tool, b experimental setup deployed [17]

advantages. This is because the rotation of the multi-hole electrode upgrades the flushing effectiveness by serving a better getaway of gaseous dielectric and eroded particles from the discharge gap, hence machining performance enhanced. Use of such an electrode prevents the formation of gudgeons in the center of the hole, during a pure drilling operation. The multi-hole tool was shown in Fig. $2 \mathrm{a}$. Figure $2 \mathrm{~b}$ shows the set up used during the experimentation process for present work.

\subsection{Description of the experiment process}

The compressed helium gas is supplied through the rotating multi-hole tool and experimentation was conducted on die-sinking EDM machine. For a specific set of input factors, three experiments corresponding to each machining period were performed and the mean MRR values are shown in Fig. 3. It has been inferred, based on the information obtained from this calculation, that the experiments must be carried out for at least 9-12 min before stable cutting conditions can be established. This explains the sharp increase in MRR as the machining time increases from 3 to $9 \mathrm{~min}$. The increase in MRR is much less steep for machining times longer than $12 \mathrm{~min}$. Based on the facts of Fig. 3, the machining time for each experiment was fixed at $15 \mathrm{~min}$. Five process variables, i.e., pulse-on time, peak current, duty cycle, electrode rotation, and discharge gas pressure, were selected for experimental work. The range of machining variables used throughout the study is detailed in Table 2. The level of each process factors was decided on the basis of the capacity of the machine and the



Fig. 3 Variation in MRR with time of machining

Table 2 Procedure parameters with ranges

\begin{tabular}{ll}
\hline Process parameters & Range \\
\hline Peak current $\left(I_{\mathrm{p}}\right)(A)$ & $3,4,5,6,7$ \\
Pulse duration $\left(T_{\text {on }}\right)(\mu \mathrm{s})$ & $100,200,300,400,500$ \\
Duty cycle $(\mathrm{DC})$ & $0.52,0.58,0.64,0.70,0.76$ \\
Tool speed $(\mathrm{rpm})$ & $200,400,600,800,1000$ \\
Discharge gas pressure $(\mathrm{GP})(\mathrm{mmHg})$ & $4,8,12,16,20$ \\
\hline
\end{tabular}

preparatory experimentation was carried out. When the current was held under $3 \mathrm{~A}$, it had been witnessed that the MRR was negligible and if more than 7 A current was chosen, it culminated in a poor surface finish requiring the assortment of the intermediate values. The range 
specified for the pulse-on time is generally used for the EDM. The framework selected for the duty cycle covers a broad range of duty cycles. Spindle rotation contributes to improved flushing efficiency. Quite high speed, though, also reduces performance. The range chosen is thus $100-900 \mathrm{rpm}$. The outward flow velocity of the air forces the eroded matter out of the discharge area. When air inlet pressure was maintained less than $3 \mathrm{mmHg}$, MRR was found to be negligible and when air inlet pressure was maintained above $15 \mathrm{mmHg}$, an explosive burst was witnessed. The air inlet pressure level therefore remained between 3 and $15 \mathrm{mmHg}$. Similar conclusions were mentioned by Singh et al. [21], Beravala and Pandey [22], and Srivastava and Pandey [23]. In this work during machining, the tool was immersed in kerosene dielectric and compressed air was provided in the gap directly through a multi-hole rotary tool. The air through a multi-hole tool is provided in the electrodes gap to analyze the effect of airflow on die-sinking EDM functioning.

In this analysis, the central composite design (CCD) is used to plan the experiments. Each experiment is carried out three times and its average is considered as a response. The experimental results for MRR and SR during GAEDM for various parametric combinations are shown in Table 3. Most commonly utilized cleaning agent acetone was applied to clean work sample. Surface analyzer (Mitutoyo SJ-210) was deployed to gauge the surface roughness of the specimens processed by GAEDM process. For accurate measurement of machining time, a digital clock with a pinpoint accuracy of $0.1 \mathrm{~s}$ has been used.

MRR is manifested as the ratio of the workpiece erosion weight to the machining time as stated below [24]:
Table 3 Experimental data of MRR and SR during GAEDM

\begin{tabular}{|c|c|c|c|c|c|c|c|}
\hline Exp. No & I & Ton & $\mathrm{DC}$ & RPM & GP & $\begin{array}{l}\text { MRR } \\
(\mathrm{mg} / \mathrm{min})\end{array}$ & $\begin{array}{l}\text { SR } \\
(\mu \mathrm{m})\end{array}$ \\
\hline 1 & 4 & 400 & 0.7 & 400 & 16 & 5.85 & 2.92 \\
\hline 2 & 4 & 400 & 0.7 & 800 & 8 & 5.9 & 3.49 \\
\hline 3 & 4 & 400 & 0.58 & 400 & 8 & 5.95 & 3.62 \\
\hline 4 & 3 & 300 & 0.64 & 600 & 12 & 6.35 & 3.7 \\
\hline 5 & 4 & 400 & 0.58 & 800 & 16 & 6.57 & 3.72 \\
\hline 6 & 5 & 500 & 0.64 & 600 & 12 & 6.92 & 3.75 \\
\hline 7 & 4 & 200 & 0.58 & 800 & 8 & 7.28 & 3.76 \\
\hline 8 & 5 & 300 & 0.64 & 1000 & 12 & 8.43 & 3.76 \\
\hline 9 & 5 & 300 & 0.64 & 600 & 4 & 8.71 & 3.84 \\
\hline 10 & 4 & 200 & 0.7 & 400 & 8 & 8.77 & 3.84 \\
\hline 11 & 6 & 400 & 0.58 & 800 & 8 & 9.7 & 3.91 \\
\hline 12 & 5 & 300 & 0.52 & 600 & 12 & 9.88 & 4.02 \\
\hline 13 & 4 & 200 & 0.58 & 400 & 16 & 10.15 & 4.02 \\
\hline 14 & 5 & 300 & 0.64 & 200 & 12 & 10.3 & 4.02 \\
\hline 15 & 5 & 300 & 0.64 & 600 & 12 & 10.45 & 4.04 \\
\hline 16 & 5 & 300 & 0.64 & 600 & 12 & 10.5 & 4.07 \\
\hline 17 & 5 & 300 & 0.64 & 600 & 12 & 10.9 & 4.14 \\
\hline 18 & 5 & 300 & 0.64 & 600 & 12 & 11.3 & 4.19 \\
\hline 19 & 5 & 300 & 0.64 & 600 & 12 & 11.6 & 4.21 \\
\hline 20 & 4 & 200 & 0.7 & 800 & 16 & 11.68 & 4.25 \\
\hline 21 & 5 & 300 & 0.64 & 600 & 12 & 11.8 & 4.26 \\
\hline 22 & 6 & 400 & 0.7 & 800 & 16 & 12.12 & 4.29 \\
\hline 23 & 6 & 400 & 0.7 & 400 & 8 & 12.96 & 4.32 \\
\hline 24 & 5 & 300 & 0.76 & 600 & 12 & 13.45 & 4.32 \\
\hline 25 & 5 & 300 & 0.64 & 600 & 20 & 13.8 & 4.37 \\
\hline 26 & 6 & 200 & 0.58 & 400 & 8 & 14.44 & 4.41 \\
\hline 27 & 6 & 400 & 0.58 & 400 & 16 & 14.46 & 4.53 \\
\hline 28 & 5 & 100 & 0.64 & 600 & 12 & 16.35 & 4.56 \\
\hline 29 & 6 & 200 & 0.7 & 800 & 8 & 17 & 4.57 \\
\hline 30 & 6 & 200 & 0.58 & 800 & 16 & 18.31 & 4.64 \\
\hline 31 & 6 & 200 & 0.7 & 400 & 16 & 19.4 & 4.84 \\
\hline 32 & 7 & 300 & 0.64 & 600 & 12 & 21.95 & 4.96 \\
\hline
\end{tabular}


Material removalrate $(\mathrm{mg} / \mathrm{min})=\frac{\text { Weight of eroded workpiece }}{\text { Machining time }}$

\section{Results and discussion}

\subsection{ANFIS analysis of MRR and SR in GAEDM}

In this work, the ANFIS strategy was applied to develop the relation between process parameters (including electrical as well as non-electrical factors) and significant EDM response factors, such as the MRR and the SR during the GAEDM. The ANFIS is a hybrid technique; hybridization is done to improve the multi-functionality of the developed model. The requirement for supplanting these essential capacities is to expand the implementation speed and upgrade the accuracy. An exceedingly intricate and not well characterized scientific framework can be modeled with ANFIS [25]. The ANFIS framework used in this study consists of five inputs as well as five layers. The Sugeno fuzzy system was used as FIS in this framework. To illustrate the ANFIS operation, the FIS was deemed to contain five inputs and one output. The fuzzy rules adopted in this work can be found in details of reference [26]. In the analysis, following the rule base, an assumption was made while applying the Sugeno approach to the numeric values acquired from the input parameters (refer Fig. 4). The model was developed after it defined the membership function and rules for inputs and outputs. Figure 5 reveals that in the first layer of the proposed system, there have been five inputs, and that in the second layer, there is a hidden layer wherein three neurons referring to the input membership functions and actually shows that there will only be single output. The simulation of GAEDM responses by the ANFIS approach consists of major stages, including training and testing. If the occurrence of ANFIS analysis will proceed, it is crucial that the entire input parameters must not be qualitative. According to the available experimental strategy, out of a maximum of 32 data, 24 data were picked at random for the training of the ANFIS program. The development of an appropriate ANFIS paradigm depends on the nature of the fuzzy theory and the form of membership structures. In this study, the fuzzy law and membership functions are used for the creation of a prediction model as shown in Table 4. Table 4 shows the ANFIS parameters in which the basis of stopping of the algorithm only to muster the number of iterations. Throughout the ANFIS, the feeds of each layer are obtained by the nodes of the previous layer. Details about ANFIS can be found in Ref. [27]. Figure 6 displays a graphical overview of the fuzzy logic rationale for gas-assisted EDM machining effects by using Matlab software, wherein rows depict the 10 rules and columns display the five-input/one-output parameters. Figure 7 shows that there is a significant correlation



System EDM-ANFIS: 5 inputs, 1 outputs, 10 rules

Fig. 4 Sugeno FIS model

SN Applied Sciences 


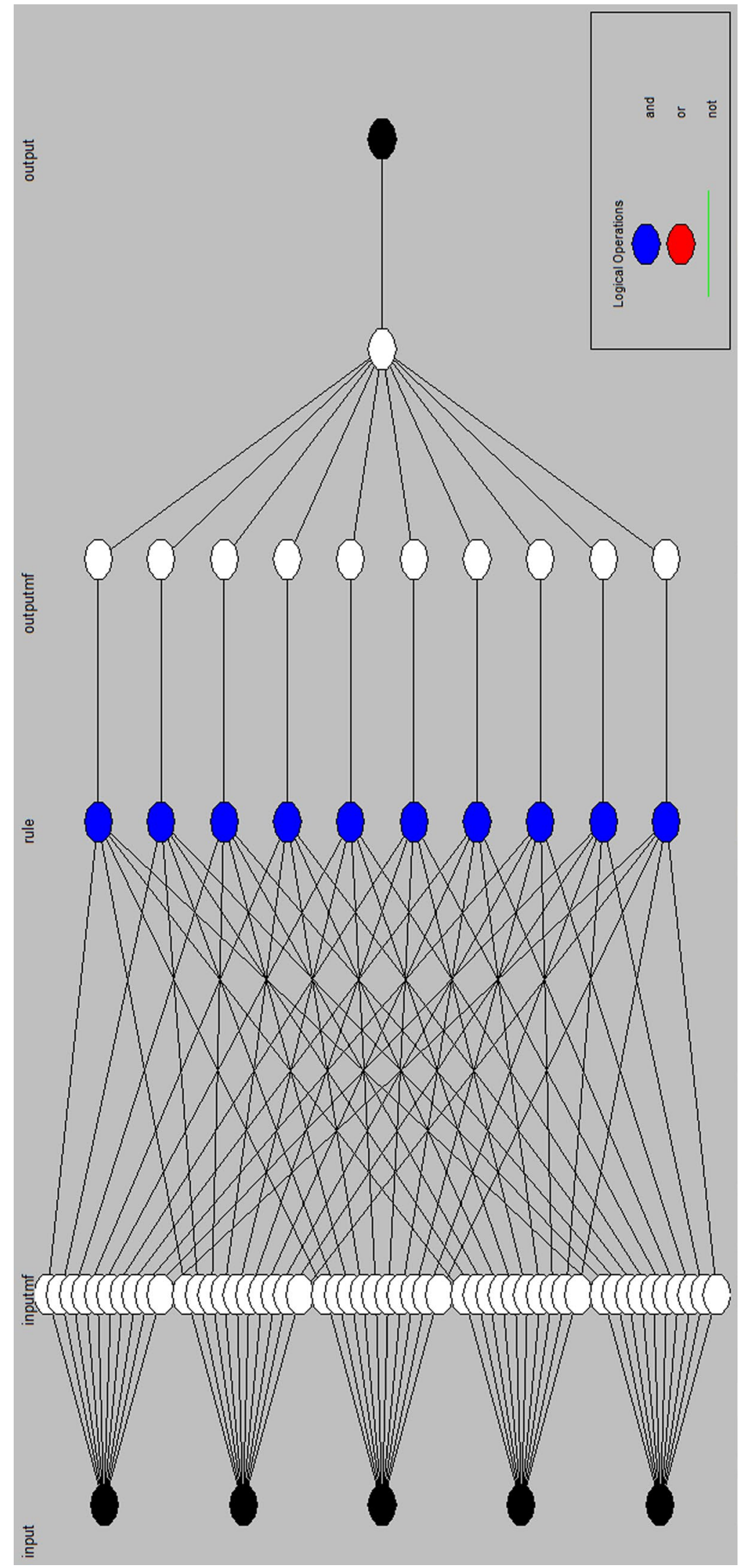

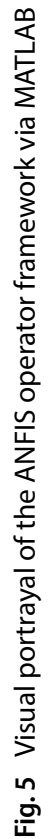


Table 4 ANFIS attributes

\begin{tabular}{ll}
\hline Nodes & 524 \\
Linear parameters & 1458 \\
Total number of parameters & 1503 \\
Training data pairs & 24 \\
Checking data pairs & 8 \\
Fuzzy rules in number & 243 \\
Membership function & Triangular \\
\hline
\end{tabular}

between the experimental estimates of the related EDM process output and also the expected values of the ANFIS model. The proposed models could be claimed to have accurately projected the assessment of the EDM response (Refer Fig. 7).

\subsection{ANFIS-GA and ANFIS-PSO}

GA and PSO are used to adjust the ANFIS as well as to achieve the optimal values of the ANFIS variables to assess the GAEDM response with accuracy and precision. In the ANFIS system, the variables are usually calibrated to the least square error technique. The effects of this technique may also be trapped in the localized optima [28]. Therefore, the GA randomized selection algorithm is considered to be the most plausible solution to the elimination of this fundamental flaw. GA comprises three key stages namely (a) activation of the population, (b) GA operators, and (c) assessment. Fitness function is used to calculate the target condition and performance $[16,29]$. The issue's fitness function was determined considering the MSE between the actual and anticipated values. The fitness function may be assessed by using Eq. 2 .

Fitness function $=1 /(1+\mathrm{MSE})$

Membership functions are linked to the previous parameters. The adaptive algorithm (ANFIS-GA) is utilized to improve the underlying factors. The factors utilized in the GA algorithm are depicted in Table 5. A code to yield the ANFIS-GA model was created in MAT LAB software. The GA-based hybrid model maintained a comparable relation between input and output parameters. PSO is a classic stochastic optimization technique. PSO algorithm is established on the conduct of swarm such as schooling of fishes or flocking of birds. Each member of a swarm works in an identical way and has a dispersed perceptiveness about finding a better solution. In PSO optimization started with a randomly chosen particle in the span of exploration and eventually searches for optimum by improving generations repetitively [30]. The main characteristics of each member of a swarm are its position and velocity. Each particle is modified as two special particles. First, the particles are the very best solution found by the particles up to this point. This is the best that has been done so far among the entire population of particles. The methodology of regression analysis is used for evaluation of each particle. When the fitness rate of the perfect particle meets the stop criteria, the program must be halted, and the variables generated should be exported. The factors utilized in the PSO algorithm are depicted in Table 6. The details of the PSO algorithm can be found elsewhere [25]. In addition, a code was also created in the MAT LAB software to yield the ANFIS-PSO model. The variables for ANFIS-GA and ANFIS-PSO were based on a combination of a systematic review, tests, and errors, as well as the most suitable values for this scenario. Here, according to the accessible thirty-two experimental results, a maximum number of twenty-four data were chosen for the ANFISGA and ANFIS-PSO algorithm training. The additional eight data which did not apply for learning were utilized during the testing of hybrid models. The flow chart of GA and PSO algorithms is demonstrated in Fig. 8. Evolutionary algorithms such as ANFIS-GA and ANFIS-PSO are designed to extract the advantages of every one of the two methods so that the model can be implemented very effectively. Histograms demonstrate the training and testing performance discrepancies from the exploratory and expected MRR estimates of the ANFIS, ANFIS-GA, and ANFIS-PSO models in Fig. 9. Likewise, the errors in the training and testing of the experimental data and the projected estimates for the SR of the ANFIS, ANFIS-GA, and ANFIS-PSO models are included in the histograms in Fig. 10.

\subsection{Comparative study of outcome anticipated by ANFIS, ANFIS-GA, and ANFIS-PSO}

Predictive model reliability was assessed using the mean average error (MAE), the root mean square error (RMSE), and the coefficient of determination (R2) $[25,28]$. The below-mentioned equations might be utilized to acquire the MAE, RMSE, and $R^{2}$.

$\mathrm{MAE}=\frac{\sum_{i=1}^{N}\left|E_{i}-P_{i}\right|}{N}$

$\mathrm{RMSE}=\sqrt{\frac{1}{N} \sum_{i=1}^{N}\left(E_{i}-P_{i}\right)^{2}}$

$R^{2}=1-\frac{\sum_{i=1}^{N}\left(E_{i}-P_{i}\right)}{\sum_{i=1}^{N}\left(E_{i}-P_{m}\right)}$ 

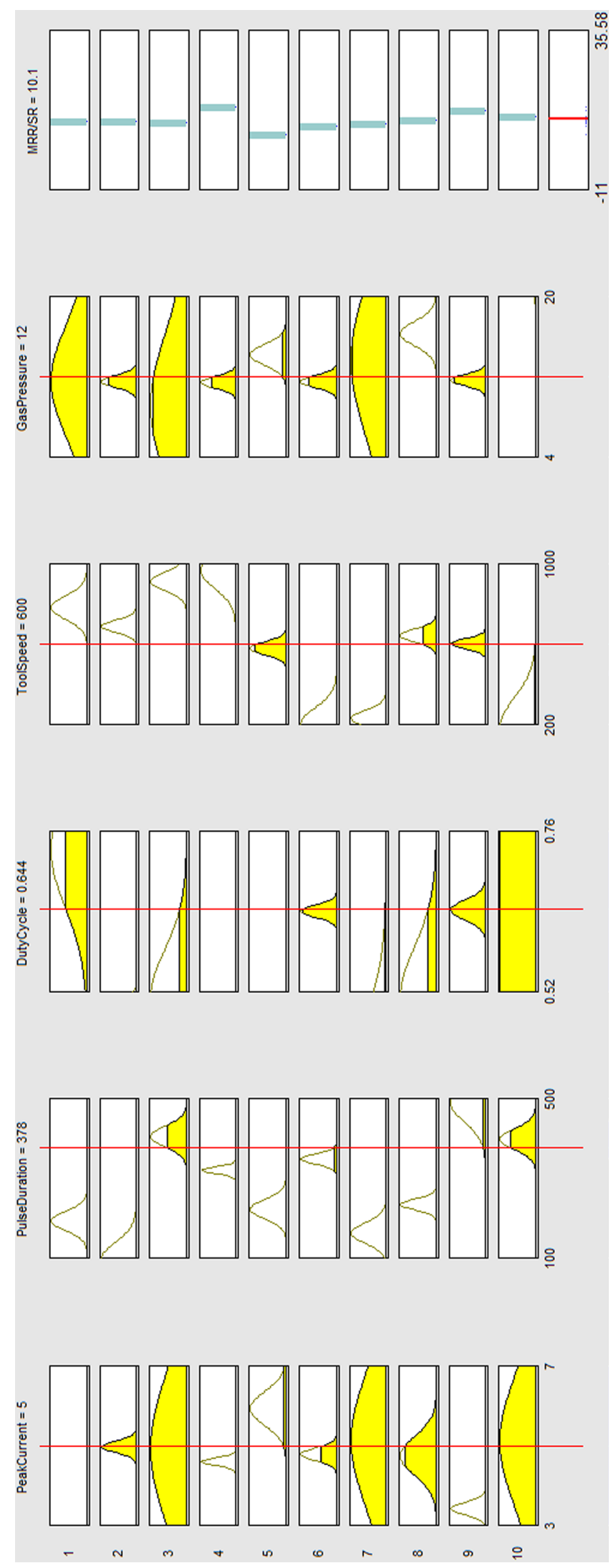

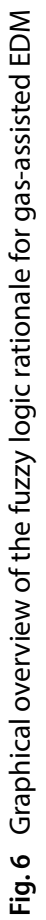



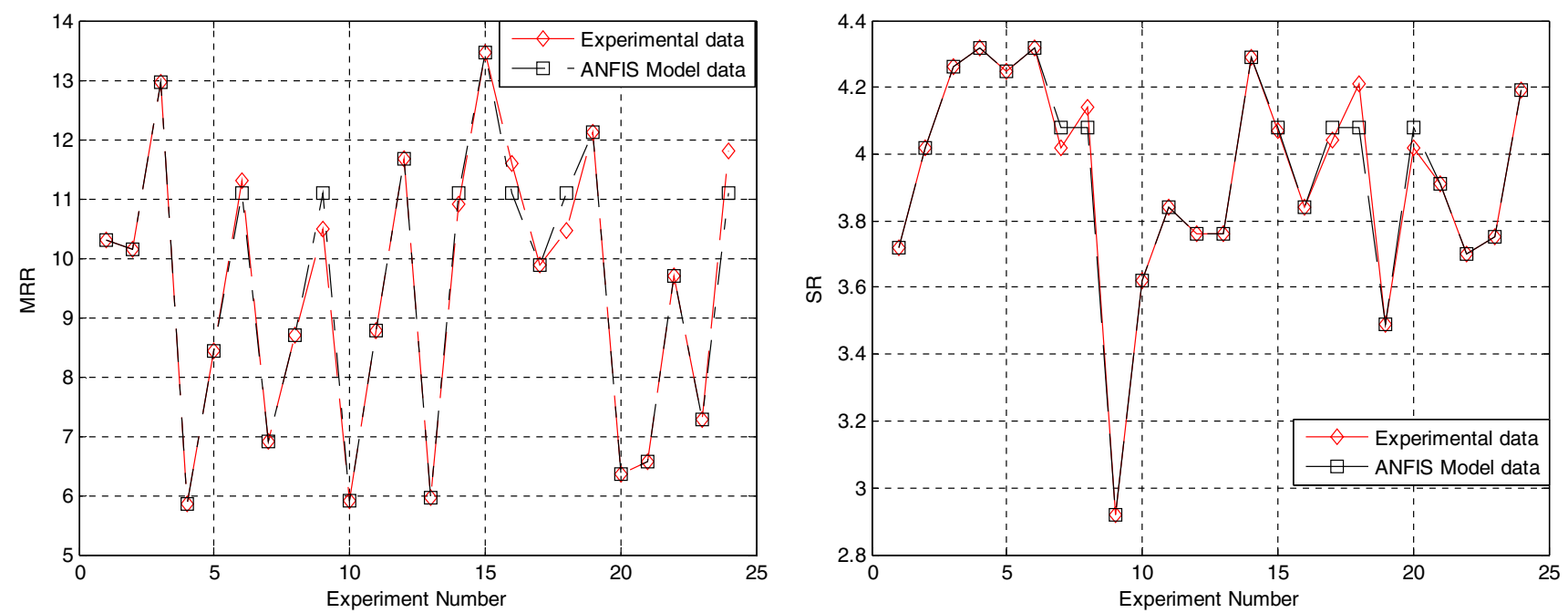

Fig. 7 Experimental and anticipated estimation by ANFIS for a MRR $\mathbf{b}$ SR

Table 5 GA algorithm parameters

\begin{tabular}{ll}
\hline GA parameters & \\
\hline Size of population & 20 \\
Number of Iteration & 2000 \\
Crossover (\%) & 0.8 \\
Mutation (\%) & 0.8 \\
Rate of mutation & 0.01 \\
Selection pressure & 8 \\
Gamma & 0.7 \\
\hline
\end{tabular}

Table 6 PSO algorithm parameters

PSO parameters

Size of population 25

Number of iteration 1000

Inertia weight

Inertia weight/damping ratio

Personal learning Coefficient

Global learning coefficient

where $P_{\mathrm{m}}=\frac{\sum_{i=1}^{N} P_{i}}{N}, N=$ Available training data, $E_{i}=$ Value of experiment data, $P_{i}=$ Anticipated values by predictive models, $P_{\mathrm{m}}=$ Mean of the anticipated values by the predictive models.

A comparative study of the expected response estimates of the ANFIS, ANFIS-GA, and ANFIS-PSO models and the approximate values compared to the respective experiment of the GAEDM method for significant EDM responses is shown in Figs. 11 and 12, respectively. The statistical analysis has been performed to assess the efficiency and effectiveness of the established ANFIS, ANFIS-GA, and ANFIS-PSO

models. The accuracy of the anticipation models has been evaluated by the statistical techniques and is shown in Tables 7 and 8 for the EDM process output. A benchmarking tool could have a significant level of accuracy when the value of MAE and RMSE is near to zero and $R^{2}$ is close to one. The comparative analysis shows that all three developed models offer a stable prediction. Tables 7 and show that the GAEDM output projected by the ANFIS-PSO model is closer to the experimental value in comparison with the ANFIS and ANFIS-GA outputs. From the value mentioned in Tables 7 and 8, it may be presumed very well that the ANFISPSO model entitle progressively accurate and correct forecast as compared to ANFIS and ANFIS-GA models.

The scatter diagrams between the experiment and the predicted outcome of considered responses are performed for the developed models for more exemplification as shown in Figs. 13 and 14 and apparently had fewer deceptions. In this way, it can very well be argued that the models developed are correct and true in the projections of significant EDM responses. Moreover, from the figure (Refer Figs. 13 and 14), the variance between the experiment and the expected values for the ANFIS-PSO framework is stated to be below the ANFIS and ANFIS-GA frameworks. The predictability of the ANFIS-GA model is better than the ANFIS model. Nevertheless, the ANFIS-PSO model is more effective in forecasting than the ANFIS and ANFIS-GA models.

\section{Conclusions}

The current study provides knowledge of superior predictive precision in the operation of the EDM. Apart from this, a better point of view is recommended to model 

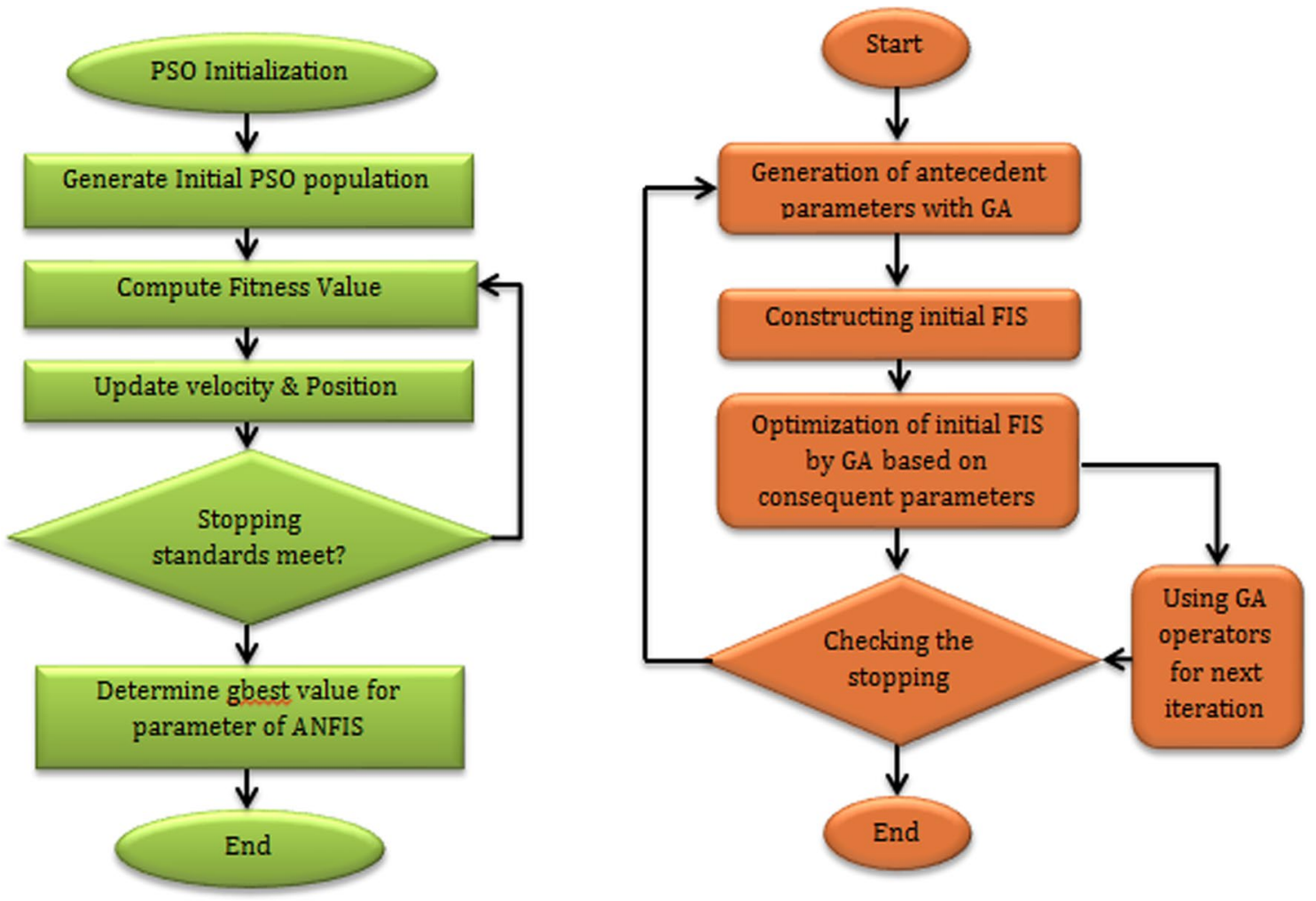

Fig. 8 Flow chart of a ANFIS-PSO, b ANFIS-GA
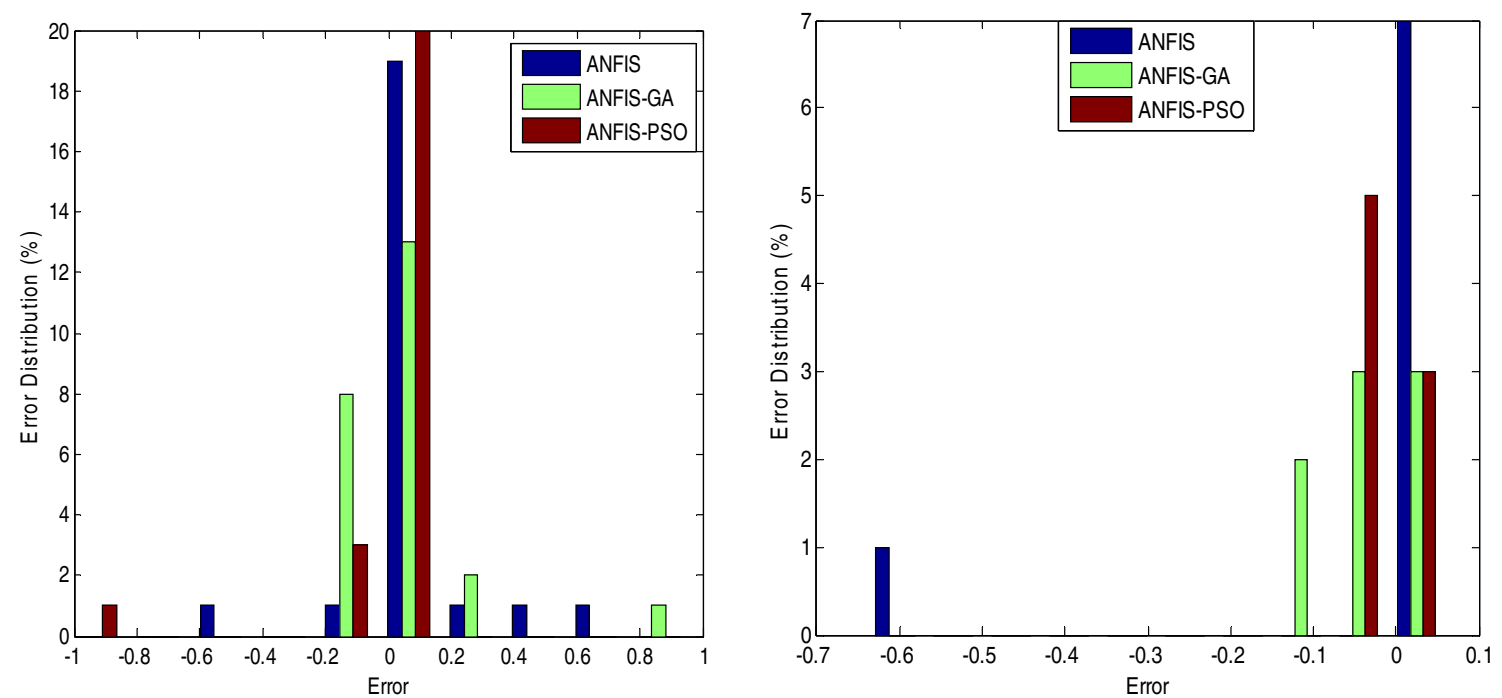

Fig. 9 Histogram of errors of MRR for $\mathbf{a}$ training, $\mathbf{b}$ testing

EDM responses with ANFIS, ANFIS-GA, and ANFIS-PSO techniques, utilizing RSM design of the experiment. The techniques focused on ANFIS, ANFIS-GA, and ANFIS-PSO were used to develop MRR and SR forecasting models during GAEDM. The research findings of this analysis are summarized as follows:
- Hybrid computing methodology incorporating the features of the ANFIS architecture and the GA and PSO algorithms have been effectively used to anticipate EDM outputs like those of MRR and SR. The MAE, RMSE, and $R^{2}$ of the ANFIS-GA and ANFIS-PSO models were observed to be smaller than the ANFIS model. 

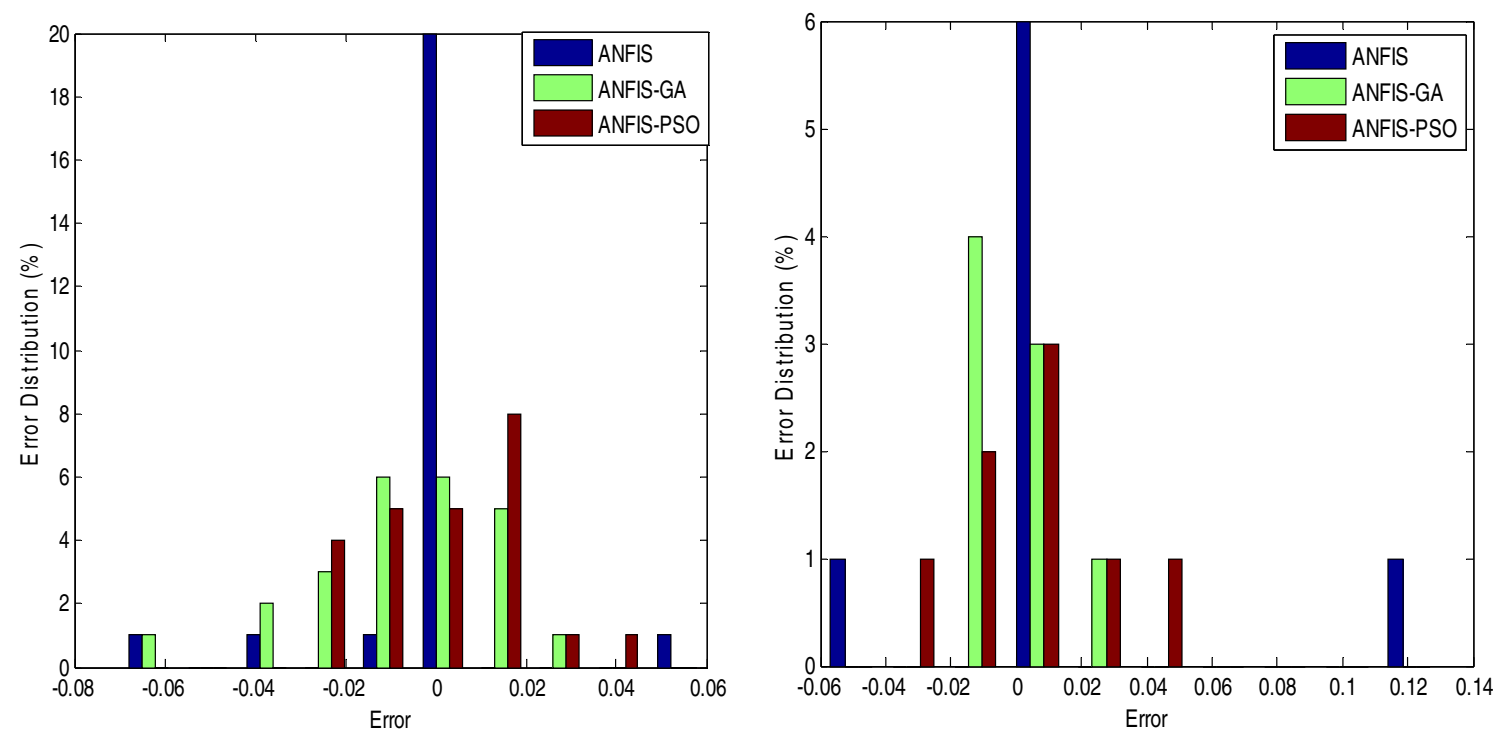

Fig. 10 Histogram of errors of SR for $\mathbf{a}$ training, $\mathbf{b}$ testing



Fig. 11 Comparative study of experimental and anticipated value of MRR by developed models

Moreover, the reliability investigation reveals that the ANFIS-PSO models show a significant improvement in accuracy, especially when compared with the ANFIS and ANFIS-GA models.

- A comparative study was conducted on each of the models designed to identify the most accurate of the



Fig. 12 Comparative study of experimental and anticipated value of SR by developed models

three models produced. The ANFIS-GA-based model provides superior prediction in comparison with the ANFIS model all in all. Moreover, ANFIS-PSO model was seen to foresee process output most factual when compared with ANFIS and ANFIS-GA models.

- The proposed models can be used to foresee additional performance of EDM process, and other process out- 
Table 7 Comparative study of various MRR models

\begin{tabular}{|c|c|c|c|c|c|c|}
\hline \multirow{2}{*}{$\begin{array}{l}\text { Model } \\
\text { Analysis }\end{array}$} & \multicolumn{2}{|l|}{ ANFIS } & \multicolumn{2}{|l|}{ ANFIS-GA } & \multicolumn{2}{|c|}{ ANFIS-PSO } \\
\hline & Training & Testing & Training & Testing & Training & Testing \\
\hline AME & 0.0921 & 0.8 & 0.1038 & 0.0637 & 0.0771 & 0.0362 \\
\hline RMSE & 0.2229 & 0.2263 & 0.222 & 0.0796 & 0.217 & 0.0417 \\
\hline$R^{2}$ & 0.966 & 0.9807 & 0.963 & 0.9848 & 0.9732 & 0.9914 \\
\hline
\end{tabular}

Table 8 Comparative study of various SR models

\begin{tabular}{|c|c|c|c|c|c|c|}
\hline \multirow{2}{*}{$\begin{array}{l}\text { Model } \\
\text { Analysis }\end{array}$} & \multicolumn{2}{|l|}{ ANN } & \multicolumn{2}{|l|}{ ANFIS-GA } & \multicolumn{2}{|c|}{ ANFIS-PSO } \\
\hline & Training & Testing & Training & Testing & Training & Testing \\
\hline AME & 0.0233 & 0.0325 & 0.0158 & 0.0225 & 0.0121 & 0.0113 \\
\hline RMSE & 0.0348 & 0.0387 & 0.0227 & 0.0255 & 0.0151 & 0.0146 \\
\hline$R^{2}$ & 0.9354 & 0.8284 & 0.956 & 0.8808 & 0.9663 & 0.9418 \\
\hline
\end{tabular}

Fig. 13 Scatter plots of experiment and anticipated estimates for MRR

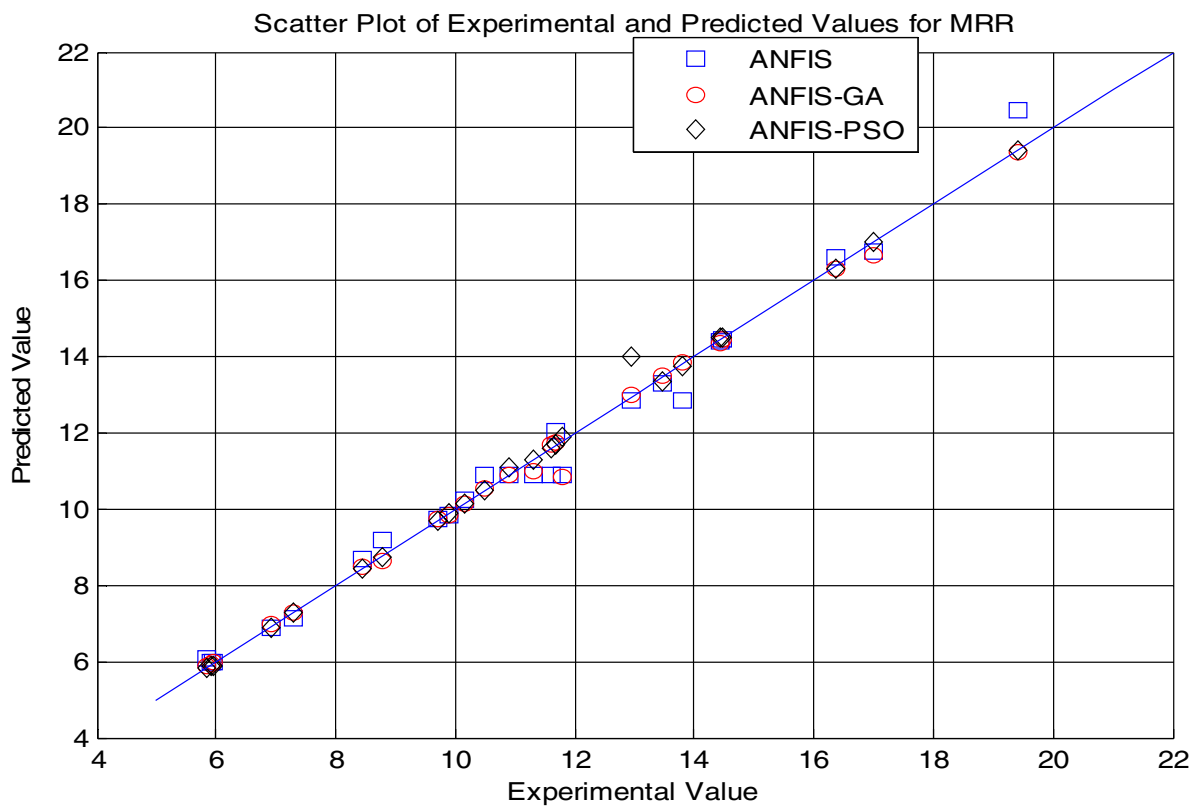

puts. In particular, the assertion of this work reveals that a model such as ANFIS-PSO is an effective and reliable approach to accurately predicting EDM responses.

- Such methods are assumed to be relevant to optimize several other related production processes. With that kind of degree of precision, any practicing engineer who wants to get quick responses for smart on-line control and optimization can use the proposed models. 
Fig. 14 Scatter plots of experiment and anticipated estimates for SR



Funding The author(s) didn't receive any monetary support for the research and publication of this paper.

\section{Compliance with ethical standards}

Conflict of interest The authors proclaim that they do not have potential conflicts of interest.

\section{References}

1. Ho KH, Newman ST (2003) State of the art electrical discharge machining (EDM). Int J Mach Tools Manuf 43:1287-1300

2. Singh NK, Pandey PM, Singh KK, Sharma MK (2016) Steps towards green manufacturing through EDM process: a review. Cogent Eng 3:1272662

3. Mandal D, Surjya KP, Saha P (2007) Modeling of electrical discharge machining process using back propagation neural network and multi-objective optimization using non-dominating sorting genetic algorithm-II. J Mater Process Technol 186:154-162

4. Assarzadeh S, Ghoreishi M (2008) Neural-network-based modeling and optimization of the electro-discharge machining process. Int J Adv Manuf Technol 39:488-500

5. Pradhan MK, Das R, Biswas CK (2009) Comparisons of neural network models on surface roughness in electrical discharge machining. Proc IMechE Part B 223:801-808

6. Patowari PK, Saha P, Mishra PK (2009) Artificial neural network model in surface modification by EDM using tungsten-copper powder metallurgy sintered electrodes. Int J Adv Manuf Technol 51:627-638

7. Panda DK (2010) Modelling and optimization of multiple process attributes of electro discharge machining process by using a new hybrid approach of neuro-grey modeling. Mater Manuf Process 25:450-461
8. Kumar S, Batish S, Singh R, Singh TP (2014) A hybrid Taguchiartificial neural network approach to predict surface roughness during electric discharge machining of titanium alloys. $J$ Mech Sci Technol 28(7):2831-2844

9. Kumar S, Choudhury SK (2007) Prediction of wear and surface roughness in electro-discharge diamond grinding. J Mater Process Technol 191:206-209

10. Agrawal SS, Yadava V (2013) Modeling and prediction of material removal rate and surface roughness in surface-electrical discharge diamond grinding process of metal matrix composites. Mater Manuf Process 28:381-389

11. Prabhu S, Uma M, Vinayagam BK (2013) Adaptive neuro-fuzzy interference system modelling of carbon nano tube-based electrical discharge machining process. J Braz Soc Mech Sci Eng. https://doi.org/10.1007/s40430-013-0047-5

12. Kar S, Chakraborty S, Dey V, Ghosh SK (2017) Optimization of surface roughness parameters of Al-6351 alloy in EDC process: a taguchi coupled fuzzy logic approach. J Inst Eng India C. https://doi.org/10.1007/s40032-016-0297-y

13. Unune DR, Mali HS (2015) Artificial neural network-based and response surface methodology-based predictive models for material removal rate and surface roughness during electrodischarge diamond grinding of Inconel 718. Proc IMechE Part B. https://doi.org/10.1177/0954405415619347

14. Prakash C, Kansal HK, Pabla BS, Puri S (2016) Multi-objective optimization of powder mixed electric discharge machining parameters for fabrication of biocompatible layer on $\beta$-Ti alloy using NSGA-II coupled with Taguchi based response surface methodology. J Mech Sci Technol 30(9):4195-4204

15. Srivastava A, Dubey AK, Shrivastava PK (2012) Computeraided hybrid ANN-GA approach for modelling and optimization of EDDG process. Int J Abras Technol 5:3-10. https://doi. org/10.1504/IJAT.2016.078294

16. Majumder H, Maity K (2018) Application of GRNN and multivariate hybrid approach to predict and optimize WEDM responses for Ni-Ti shape memory alloy. Appl Soft Comput 70:665-679

17. Singh J, Sharma RK (2016) Implementation of Taguchi method with hybrid decision making tools for prediction of 
surface characteristics for powder-mixed EDM of WC. Persp Sci 8:455-458

18. Fard RK, Afza RA, Teimouri R (2013) Experimental investigation, intelligent modeling and multi-characteristics optimization of dry WEDM process of Al-SiC metal matrix composite. J Manuf Process 15(4):483-494

19. Roy T, Dutta RK (2018) Integrated fuzzy AHP and fuzzy TOPSIS methods for multi-objective optimization of electro discharge machining process. Soft Comput. https://doi.org/10.1007/s0050 0-018-3173-2

20. Singh NK, Singh Y, Kumar S, Sharma A (2019) Comparative study of statistical and soft computing-based predictive models for material removal rate and surface roughness during helium-assisted EDM of D3 die steel. SN Appl Sci. https://doi. org/10.1007/s42452-019-0545-x

21. Jang JSR (1993) ANFIS: adaptive-network-based fuzzy inference system. IEEE Trans Syst Man Cybern 23:665-685

22. Srivastava V, Pandey PM (2012) Effect of process parameters on the performances of EDM process with ultrasonic assisted cryogenically cooled electrode. J Manuf Process 14:393-402

23. Singh NK, Singh Y, Kumar S et al (2020) Integration of GA and neuro-fuzzy approaches for the predictive analysis of gas-assisted EDM responses. SN Appl Sci 2:137. https://doi. org/10.1007/s42452-019-1533

24. Singh NK, Pandey PM, Singh KK (2016) EDM with air assisted multi-hole rotating tool. Mater Manuf Process 31(14):1872-1878
25. Pradhan MK, Biswas CK (2010) Neuro-fuzzy and neural networkbased prediction of various responses in electrical discharge machining of AISI D2 steel. Int J Adv Manuf Technol 50:591-610

26. Pandey RK, Panda SS (2014) Optimization of bone drilling parameters using grey-based fuzzy algorithm. Measurement 47:386-392. https://doi.org/10.1016/j.measuremen t.2013.09.007

27. Gudise VG, Venayagamoorthy GK (2003) Comparison of particle swarm optimization and backpropagation as training algorithms for neural networks. In: Proceedings of the Swarm intelligence symposium, SIS '03, 24-26 Apr 2003 IEEE

28. Beravala $\mathrm{H}$, Pandey PM (2018) Experimental investigations to evaluate the effect of magnetic field on the performance of air and argon gas assisted EDM processes. J Manuf Process 34:356-373

29. Sethuramalingam P, Sundararaj OR (2017) Adaptive neurofuzzy interference system modelling of EDM process using CNT infused copper electrode. Int J Int Engg Syst 10(3):102-116

30. Rezakazemi M, Dashti A, Asghari M, Shirazian S (2017) H2-selective mixed matrix membranes modeling using ANFIS, PSOANFIS, GA-ANFIS. Int J Hydrogen Energy 42(22):15211-15225

Publisher's Note Springer Nature remains neutral with regard to jurisdictional claims in published maps and institutional affiliations. 